\title{
Mitochondrial Division Inhibitor 1 Attenuates Mitophagy in a Rat Model of Acute Lung Injury
}

\author{
Xu Luo, ${ }^{1}$ Ruimeng Liu $\left(\mathbb{D},{ }^{2}\right.$ Zhihao Zhang $\left(\mathbb{D},{ }^{2}\right.$ Zhugui Chen $\left(\mathbb{D},{ }^{3}\right.$ \\ Jian $\mathrm{He}\left(\mathbb{D},{ }^{4}\right.$ and Youtan Liu $\left({ }^{2}{ }^{2}\right.$ \\ ${ }^{1}$ Department of Critical Care Medicine, People's Hospital of Longhua, Shenzhen 518109, China \\ ${ }^{2}$ Department of Anesthesiology, Shenzhen Hospital, Southern Medical University, Shenzhen 518110, China \\ ${ }^{3}$ Department of Anesthesiology, Affiliated Hospital of Guangdong Medical University, Zhanjiang 524023, China \\ ${ }^{4}$ Department of Anesthesiology, The First People's Hospital of Foshan, Foshan 528000, China
}

Correspondence should be addressed to Jian He; hejian2011@126.com and Youtan Liu; youtanliuhao@163.com

Received 20 January 2019; Accepted 17 April 2019; Published 8 May 2019

Academic Editor: Kota V. Ramana

Copyright (C) 2019 Xu Luo et al. This is an open access article distributed under the Creative Commons Attribution License, which permits unrestricted use, distribution, and reproduction in any medium, provided the original work is properly cited.

\begin{abstract}
The regulation of intracellular mitochondria degradation is mediated by mitophagy. While studies have shown that mitophagy can lead to mitochondrial dysfunction and cell damage, the role of Mdivi-1 and mitophagy remains unclear in acute lung injury (ALI) pathogenesis. In this study, we demonstrated that Mdivi-1, which is widely used as an inhibitor of mitophagy, ameliorated acute lung injury assessed by HE staining, pulmonary microvascular permeability assay, measurement of wet/dry weight (W/D) ratio, and oxygenation index $(\mathrm{PaO} 2 / \mathrm{FiO} 2)$ analysis. Then, the mitophagy related proteins were evaluated by western blot. The results indicated that LPS-induced activation of mitophagy was inhibited by Mdivi-1 treatment. In addition, we found that Mdivi-1 protected A549 cells against LPS-induced mitochondrial dysfunction. We also found that Mdivi-1 reduced pulmonary cell apoptosis in the LPS-challenged rats and protected pulmonary tissues from oxidative stress (represented by the content of superoxide dismutase, malondialdehyde and lipid peroxides in lung). Moreover, Mdivi-1 treatment ameliorated LPS-induced lung inflammatory response and cells recruitment. These findings indicate that Mdivi-1 mitigates LPS-induced apoptosis, oxidative stress, and inflammation in ALI, which may be associated with mitophagy inhibition. Thus, the inhibition of mitophagy may represent a potential therapy for treating ALI.
\end{abstract}

\section{Introduction}

Clinical acute lung injury (ALI) is a common complication that occurs following sepsis among ICU patients and is associated with a high morbidity and mortality $[1,2]$, for which there are currently no biological therapies [3]. LPS is a major endotoxin component of gram-negative bacteria and plays an essential role in the development of ALI [4,5]. Currently, it is thought that the multiple organ dysfunctions associated with sepsis can be attributed to a pathochemical and pathophysiological injury cascade, including the inflammatory response, macroautophagy, mitochondria dysfunction, and apoptosis $[6,7]$; moreover, oxidative stress is also involved in the pathogenesis of ALI [8].

The lysosomal machinery is used to remove dysfunctional mitochondria through selective degradation via autophagy, a process termed mitophagy. However, the role of mitophagy in the development of disease remains controversial. While some studies have confirmed that excessive mitophagy promotes mitochondrial damage in chronic obstructive pulmonary disease and excessive mitochondrial fission is related to neurodegeneration and other mitochondriopathies $[9,10]$, its role in ALI remains unknown. It has been demonstrated that mitochondrial dynamics, which are primarily regulated by the GTPase dynamin-related protein, play a crucial part in regulating mitophagy $[11,12]$. Mitophagy can be blocked using the mitochondrial division inhibitor 1 (Mdivi-1).

Thus, previous studies have shown that excessive mitophagy can cause mitochondrial damage and that Mdivi- 1 can provide protection against various pathological conditions [13]. In this study, we hypothesize that mitophagy contributes 
to the development of ALI, whereas treatment with Mdivi1 may prevent LPS-induced mitophagy and alleviate lung injury in rats. Our findings suggest that the inhibition of mitophagy may merit further exploration as a potential therapy for the treatment of ALI.

\section{Materials and Methods}

2.1. Reagents and Antibodies. MitoProbe ${ }^{\mathrm{TM}}$ JC-1 $\left(5,5^{\prime}, 6,6^{\prime}-\right.$ Tetrachloro-1,1',3,3'-tetraethyl-imidacarbocyanine iodide) were purchased from Molecular Probes (Invitrogen, CA, USA). The CellTiter-Glo ${ }^{\circledR}$ assay and a terminal deoxynucleotidyl transferase dUTP nick-end labeling (TUNEL) staining kit were supplied from Promega Corp. (Madison, WI, USA). Human lung alveolar epithelial cell lines (A549) were obtained from Guangzhou Cellcook Biotech Co., Ltd. (Guangzhou, China). Caspase 3 activity assay kit was obtained from Biovision (San Francisco, USA). Immunohistochemical kits were provided by EnVision ${ }^{\mathrm{TM}}$ (Dako, Copenhagen, Denmark). LPO kit was obtained from Cayman Chemical Co. (Ann Arbor, Michigan, USA). SOD and MDA kits were obtained from Jiancheng (Nanjing, China). LPS ( $E$. coli serotype O111:B4), Mdivi-1, and other chemicals were purchased from Sigma-Aldrich (Saint Louis, MO, USA).

2.2. Animals. Procedures involving animals and their care were approved by the Medical Faculty Ethics Committee of Southern Medical University, Guangzhou, China, and complied with the NIH Guidelines for the Care and Use of Laboratory Animals. Male Sprague-Dawley rats (weight: 180 g-220 g) were acquired from the Experimental Animal Centre of South Medical University and housed under temperatureand humidity-controlled conditions on a 12/12 h day/night cycle with unrestricted access to standard diet and tap water.

2.3. ALI Model. A rat model of ALI was induced by an intratracheal administration of LPS. Animals were anesthetized under sodium pentobarbital ( $30 \mathrm{mg} / \mathrm{kg}$ body weight) intramuscularly and then placed in a supine position. The trachea was surgically exposed, after which $5 \mathrm{~mL}$ of LPS (10 mg/kg body weight, Sigma) was slowly injected into the trachea of each rat.

2.4. Cell Culture and Stimulation. A549 cells were grown at $37^{\circ} \mathrm{C}$ in $5 \% \mathrm{CO}_{2}$ in Dulbecco's modified minimum essential medium (DMEM) containing low glucose, penicillin (100 $\mathrm{U} / \mathrm{mL}$ ), streptomycin (100 U), and 10\% fetal bovine serum. The cells were stimulated in $10 \mu \mathrm{g} / \mathrm{mL}$ LPS for $6 \mathrm{~h}$ to establish LPS-induced ALI in vitro.

2.5. Drug Treatment and Experimental Grouping. The cells were randomly divided into 4 groups ( $n=6$ per group) using a random number table: (1) control group (cells treated with vehicle and without LPS stimulation); (2) Mdivi-1 control group (cells treated with $10 \mu \mathrm{M}$ of Mdivi-1 and without LPS stimulation); (3) LPS group (cells pretreated with vehicle $60 \mathrm{~min}$, followed by LPS stimulation); (4) Mdivi-1 group (cells pretreated with $10 \mu \mathrm{M}$ of Mdivi-1 $60 \mathrm{~min}$ before LPS stimulation). DMSO was used as vehicle.
The animals were randomly divided into 4 groups ( $n=6$ per group) using a random number table: (1) control group (pretreated with $0.5 \mathrm{~mL}$ DMSO for $60 \mathrm{~min}$ via the caudal vein and given $0.5 \mathrm{~mL}$ intratracheal normal saline (NS)); (2) Mdivi-1 control group (rats pretreated with a dose of Mdivi-1 $(3 \mathrm{mg} / \mathrm{kg}$ ) dissolved in $0.5 \mathrm{~mL}$ DMSO for $60 \mathrm{~min}$ and given $0.5 \mathrm{~mL}$ intratracheal NS); (3) LPS group (rats pretreated with $0.5 \mathrm{~mL}$ DMSO for $60 \mathrm{~min}$ via the caudal vein followed by LPS instillation); (4) Mdivi-1 group (rats pretreated with a dose of Mdivi-1 (3 mg/kg) dissolved in $0.5 \mathrm{~mL}$ DMSO for $60 \mathrm{~min}$ via the caudal vein followed by LPS instillation).

2.6. Western Blot Analysis. Proteins were resolved on $10 \%$ SDS-PAGE and electroblotted onto a polyvinylidene fluoride (PVDF) membrane and blocked in 5\% skimmed milk. The membranes were incubated overnight at $4^{\circ} \mathrm{C}$ with primary antibodies (all antibodies were obtained from Abcam, Cambridge, UK) against TOM20 (1:1,000 dilution), TIM23 (1:1,000 dilution), PGC-1 $\alpha$ (1:1,000 dilution), mt-TFA (1:1,000 dilution), LC3 (1:1,000 dilution), P62 (1:1,000 dilution), F4/80 (1:1000 dilution), and GAPDH (1:5,000 dilution), respectively. Horseradish peroxidase-conjugated antirabbit IgG (1:5,000 dilution) was used as the secondary antibody. Immunoreactivity was detected with an enhanced chemiluminescence detection system (Beyotime, Haimen, China) and visualized on X-ray film (Kodak, Shanghai, China).

2.7. Measurement of Mitochondrial Function. The mitochondrial membrane potential $(\Delta \Psi \mathrm{m})$ was determined using the potential-sensitive fluorescent dye JC-1. Cells were treated and subjected to LPS. JC-1 $(5 \mu \mathrm{M})$ was loaded onto cells for 15 $\min$ at $37^{\circ} \mathrm{C}$. The results were visualized using an inverted fluorescent microscope (Nikon, Ti-E Live Cell Imaging System, Japan).

Intracellular ATP was determined by a luciferase-based assay (CellTiter-Glo ${ }^{\circledR}$, Promega, Madison, WI), according to the manufacturer's recommendation. The luminescence was recorded in an automatic microplate reader (SpectraMax M5; Molecular Devices, Sunnyvale, CA, USA).

2.8. Immunohistochemistry. Bax and Bcl-2 expression in the lungs were visualized using commercial immunohistochemistry kits. The working dilution of the antibodies against Bax and Bcl-2 was 1:200.

2.9. Oxygenation Index $\left(\mathrm{PaO}_{2} / \mathrm{FiO}_{2}\right)$ Analysis. Twelve hours following LPS administration, arterial blood was collected from the carotid artery and analyzed using a commercial blood gas analyzer (model ABL8000; Radiometer Copenhagen, Westlake, Ohio). The oxygenation index was determined as the ratio of arterial oxygen partial pressure/fractional inspired oxygen $\left(\mathrm{PaO}_{2} / \mathrm{FiO}_{2}\right)$.

2.10. HÆE Staining and Scoring. Twelve hours following the administration of LPS, the right lobes of the lungs were immersed in $10 \%$ neutral buffered formalin, fixed, paraffinembedded, and sliced. Following H\&E staining, pathological changes of the lung tissues were evaluated as in previous study [14]. 
2.11. Lung Microvascular Permeability Analysis. The permeability assay was performed as previously described [15]. A sample of the lung tissue was weighed, homogenized, and immersed in N,N-dimethylformamide (Sigma, USA). Eluted EB was measured at a wavelength of $620 \mathrm{~nm}$ using an automatic microplate reader and the amount was expressed as micrograms per $100 \mathrm{mg}$ of dry tissue.

2.12. Lung Wet/Dry (W/D) Ratio In Vivo. Following the administration of LPS, the water content of the lungs was measured. The right lungs were excised, blotted, and weighed to obtain the wet weight, then desiccated at $80^{\circ} \mathrm{C}$ for $48 \mathrm{~h}$ to obtain the dry weight. The wet/dry ratio was calculated as an assessment of tissue edema.

2.13. Assessments of Cell Apoptosis. Apoptosis was measured using terminal deoxynucleotidyl transferase-mediated dUDP nick-end labeling (TUNEL) staining. The level of caspase 3 activity was determined in the lung tissue homogenates using a caspase 3 Fluorometric Assay Kit.

2.14. Measurement of Lipid Peroxides (LPO), Superoxide Dismutase (SOD), and Malondialdehyde (MDA). The level of LPO, SOD, and MDA in lung was determined using commercial assay kits in accordance with the respective manufacturer's guidelines.

2.15. Measurement of Inflammatory Mediators in Lung and the Bronchoalveolar Lavage Fluid (BALF). BALF samples were collected by washing the lung three times with $4 \mathrm{~mL}$ PBS through a tracheal cannula placed into each rat under anesthesia. Briefly, animals were sacrificed and the chest was opened, a median sternotomy was performed, and the trachea was isolated using a blunt dissection. Next, a suitable smallcaliber tube was inserted into the airway and secured. Then PBS solution was infused slowly into the lungs, and the BALF was withdrawn into the tube. The fluid recovery rate was > $80 \%$. Lavage samples were centrifuged at $1,500 \mathrm{~g}$ for $10 \mathrm{~min}$ at $4^{\circ} \mathrm{C}$.

The concentration of TNF- $\alpha$, IL- $1 \beta$, and IL- 6 in the lung and BALF was measured using a commercial enzyme-linked immunosorbent assay kit. The results were expressed as $\mathrm{g} / \mathrm{mg}$ of tissue or $\mathrm{pg} / \mathrm{mL}$ of BALF.

2.16. Myeloperoxidase Activity Assay. Lung tissues were harvested, rinsed, homogenized, and centrifuged. Supernatants were collected and subjected to enzyme-linked immunosorbent assay for determination of myeloperoxidase (MPO) activity using the MPO activity colorimetric assay kit (Biovision, Zurich, Switzerland).

2.17. Statistical Analysis. Experimental results were presented as the mean standard error $( \pm S D)$. Statistical analysis was performed using a one-way analysis of variance (ANOVA) followed by an LSD multiple comparison test and Student's $t$-test where appropriate. A value of $\mathrm{P}<0.05$ was considered statistically significant, and $\mathrm{n}$ represents the number of animals.

\section{Results}

3.1. Mdivi-1 Treatment Attenuates Lung Injury following LPS Administration in Rats. It has been well-established that the administration of LPS causes injury to the lung. In order to assess whether Mdivi-1 treatment was actually provided a protective role against acute lung injury in rats, H\&E staining was performed on the lung tissues to observe the extent of injury within the lung. As shown in Figure 1(a), the LPS + vehicle group displayed severe inflammatory cell infiltration, thickened alveolar walls, diffuse edema, decreased alveolar space, and enhanced interstitial congestion compared to the control and vehicle groups, which were comparable. In addition, LPS exposure was also associated with an increased lung injury score and W/D ratio, as well as a decreased oxygenation index $(\mathrm{P}<0.05$ for LPS + vehicle versus control; Figures $1(\mathrm{~b})-1(\mathrm{~d}))$. These data suggest that the intratracheal administration of LPS results in significant ALI. Conversely, treatment with moderate $(3 \mathrm{mg} / \mathrm{kg})$ and high $(5 \mathrm{mg} / \mathrm{kg})$ doses of Mdivi-1 dramatically attenuated the observed histopathological changes, lung injury score, W/D ratio, and oxygenation index, whereas a small $(1 \mathrm{mg} / \mathrm{kg})$ dose could not achieve such effects $(\mathrm{P}<0.05$ for LPS + vehicle versus LPS $+3 \mathrm{mg} / \mathrm{kg}$ and LPS $+5 \mathrm{mg} / \mathrm{kg} ; \mathrm{P}>0.05$ for LPS + vehicle versus LPS + $1 \mathrm{mg} / \mathrm{kg}$; Figure 1 ). So, we selected the dose of $3 \mathrm{mg} / \mathrm{kg}$ of Mdivi-1 as the treatment in the following experiment in vivo. Moreover, we found that Mdivi-1 significantly reduced the lung microvascular permeability reflecting by the lower content of Evans Blue in the lung (Figure 6(h)). These results demonstrate that Mdivi1 treatment significantly improves the lung function in LPSchallenged rats.

\subsection{Mdivi-1 Treatment Inhibits the Activation of Mitophagy in} the Lung. Measuring the mitochondrial mass is a quantitative method of monitoring the mitophagy. The level of the mitochondrial markers, TOM20 and TIM23, was used as indicators of mitophagy activity. The immunoblot analysis revealed that the level of TOM20 and TIM23 expression decreased following LPS exposure $(\mathrm{P}<0.05$ for Control versus LPS + vehicle). In contrast, Mdivi-1 treatment inhibited the downregulation of TOM20 and TIM23 expression $(\mathrm{P}<0.05$ for LPS + vehicle versus Mdivi-1; Figures 2(b) and 2(c)). This loss of mitochondrial mass may be due to mitophagy, or alternatively, it could be caused by an overall increase in autophagy or decreased mitochondrial biogenesis. Thus, we analyzed the expression level of two general autophagy-related proteins, LC3 and p62, as well as PGC- $1 \alpha$ and mt-TFA, two mitochondrial biogenesis-related proteins. We found that the expression of PGC- $1 \alpha$ and $\mathrm{mt}-$ TFA was not significantly downregulated following Mdivi1 treatment $(\mathrm{P}>0.05$ for LPS + vehicle versus Mdivi-1; Figures 2(d) and 2(e)). Moreover, Mdivi-1 treatment did not significantly upregulate the level of LC3-II and downregulate that of P62, suggesting that autophagic flux was not robustly reinforced $(P>0.05$ for LPS + vehicle versus Mdivi-1; Figures $2(\mathrm{f})$ and $2(\mathrm{~g}))$. These data suggest that the effect of Mdivi-1 on mitochondrial mass is produced by inhibiting mitophagy. 


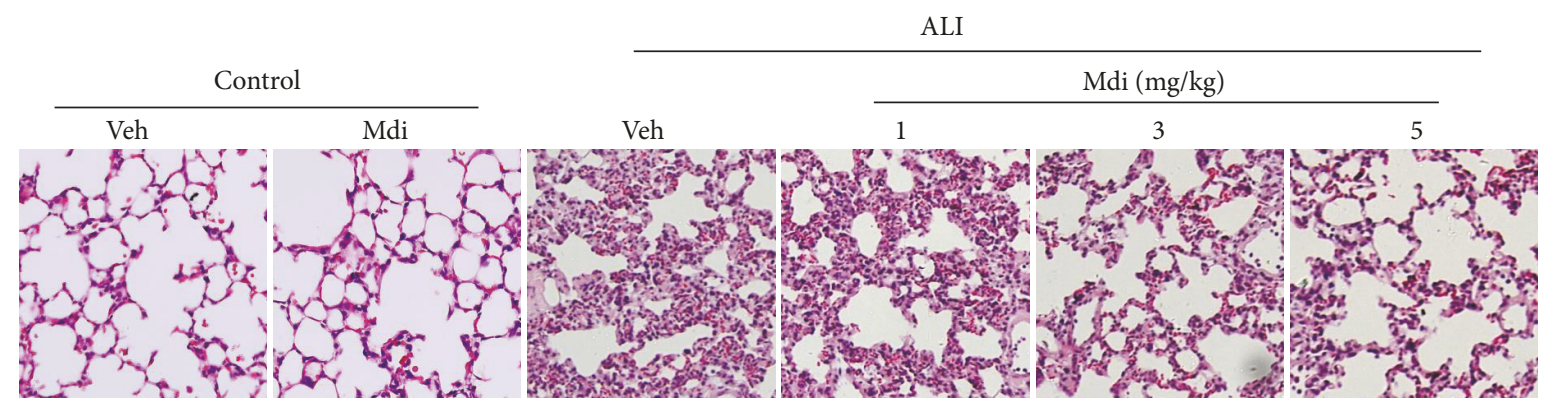

(a)

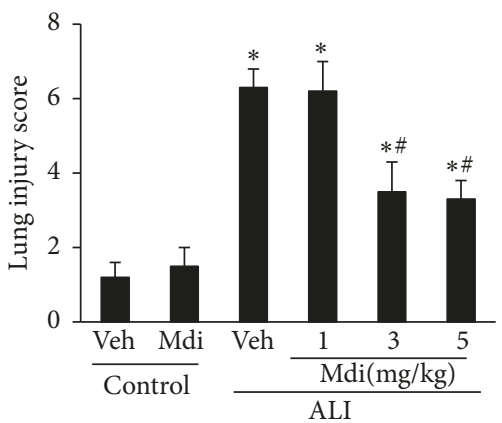

(b)

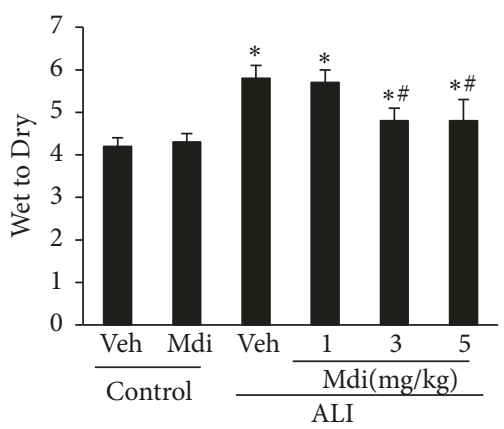

(c)

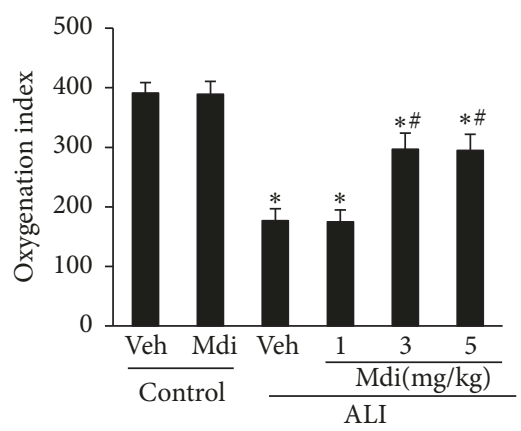

(d)

Figure 1: Mdivi-1 treatment attenuates lung histopathology and pulmonary function. (a) Comparison of the morphological changes of the lung tissues $(200 \times$ magnification). Hematoxylin and eosin staining: the LPS + vehicle group displayed severe inflammatory cell infiltration, diffuse edema with less alveolar space, and increased interstitial congestion. Moderate ( $3 \mathrm{mg} / \mathrm{kg})$ and high $(5 \mathrm{mg} / \mathrm{kg})$ doses of Mdivi-1 treatment markedly prevented the morphological changes induced by LPS administration. (b to d) Increased lung injury score and lung W/D ratio, as well as decreased $\mathrm{PaO}_{2} / \mathrm{FiO}_{2}$, were observed in the LPS + vehicle group, which were improved following both the moderate or high dose of Mdivi-1 treatment. Data are expressed as the mean $\pm \mathrm{SD}(\mathrm{n}=6$ in each group $) . * \mathrm{P}<0.05$ versus control group; $\# \mathrm{P}<0.05$ versus LPS + vehicle group.

Therefore, it was confirmed that Mdivi-1 inhibited mitophagy activation in ALI.

\subsection{Mdivi-1 Treatment Inhibits LPS-Induced Mitochondrial} Dysfunction In Vitro. To evaluate whether Mdivi-1 may act as a survival factor for lung alveolar epithelial cells, we examined the effect of Mdivi-1 on cell death induced by LPS insult. Cultured A549 cells with above-mentioned treatments, A549 cells viability significantly decreased in comparison with the control group after LPS stimulation $(\mathrm{P}<0.05)$. Pretreated with moderate $(10 \mu \mathrm{M})$ and high $(50 \mu \mathrm{M})$ doses of Mdivi-1 for $24 \mathrm{~h}$, the survival rates of A549 cells were markedly reversed, whereas a small $(1 \mu \mathrm{M})$ dose could not achieve such effects $(\mathrm{P}<0.05$ for LPS + vehicle versus LPS $+10 \mu \mathrm{M}$ and LPS +50 $\mu \mathrm{M} ; \mathrm{P}>0.05$ for LPS + vehicle versus LPS $+1 \mu \mathrm{M}$; Figure $3(\mathrm{a}))$. So, we selected the concentration of $10 \mu \mathrm{M}$ of Mdivi-1 as the treatment in the following experiment in vivo.

To characterize the role of Mdivi-1 on LPS-induced mitochondrial dysfunction in vitro, mitochondrial membrane potential $(\Delta \Psi \mathrm{m})$ and cellular ATP levels were measured using commercial kits. As shown in Figure 3(b), it was found that $\Delta \Psi \mathrm{m}$ was significantly decreased as indicated by the marked increases in green fluorescence when cells were exposed to LPS insult. However, when cells were pretreated with Mdivi-1, LPS-induced mitochondrial depolarization was significantly attenuated $(\mathrm{P}<0.05$ for LPS + vehicle versus
Mdivi-1; Figure 3(c)). Furthermore, Mdivi-1 substantially restored mitochondrial ATP levels after LPS insult $(\mathrm{P}<0.05$ for LPS + vehicle versus Mdivi-1; Figure 3(d)).

\subsection{Mdivi-1 Treatment Alleviates Lung Cell Apoptosis fol-} lowing LPS Administration in Rats. Lung cell apoptosis was increased upon LPS administration, as determined by TUNEL staining. Treatment with Mdivi-1 was able to attenuate such LPS-induced cell death $(\mathrm{P}<0.05$ for LPS + vehicle versus Mdivi-1; Figures 4(a) and 4(b)). Similarly, the level of caspase 3 activity was also found to be elevated following LPS administration but was suppressed by Mdivi-1 treatment $(\mathrm{P}<0.05$ for LPS + vehicle versus Mdivi-1; Figure 4(c)). Moreover, we examined the effect of Mdivi-1 treatment on the regulation of $\mathrm{Bcl}-2$ family protein expression. As shown in Figure 4(d), the immunohistochemical staining revealed that Mdivi-1 treatment significantly prevented the LPS-induced downregulation of $\mathrm{Bcl}-2$ and upregulation of Bax protein expression $(\mathrm{P}<0.05$ for LPS + vehicle versus Mdivi-1; Figure $4(d)$ ). Taken together, these findings indicate that Mdivi-1 protects the lung against LPS-induced apoptosis.

\subsection{Mdivi-1 Treatment Protects Oxidative Stress in the Lung.} Since oxidative stress is a leading cause of ALI pathogenesis, we investigated the level of SOD, MDA, and LPO in the lung tissues of LPS-exposed rats. As shown in Figure 5, the 


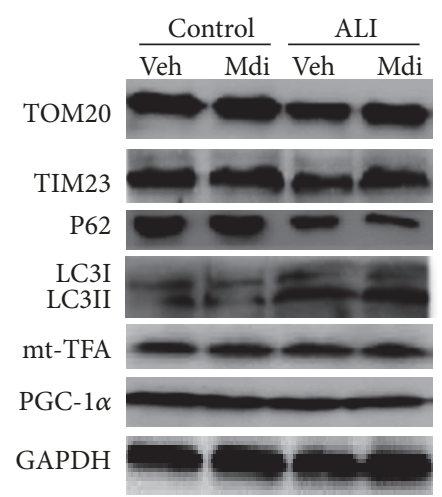

(a)

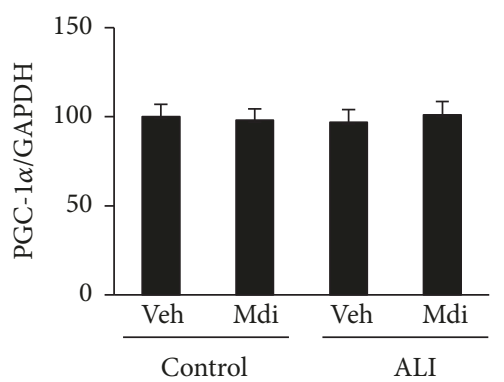

(d)

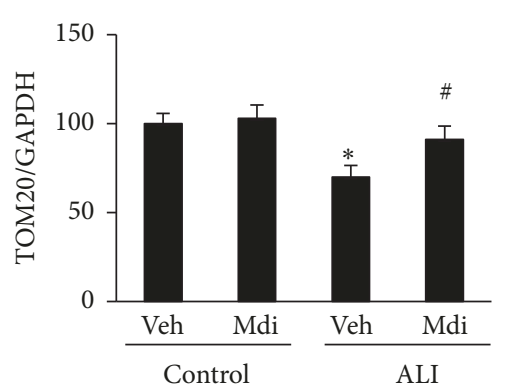

(b)

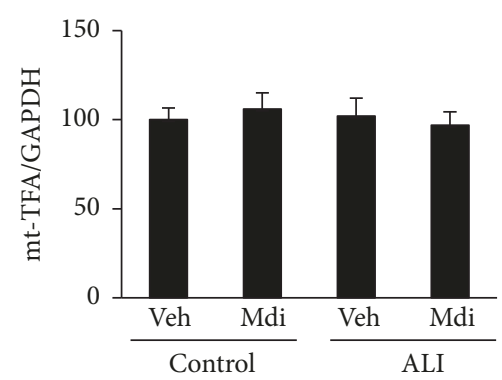

(e)

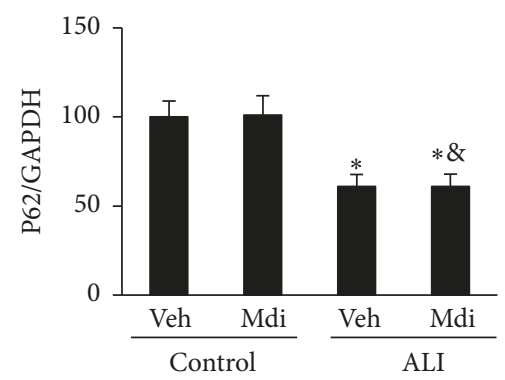

(g)

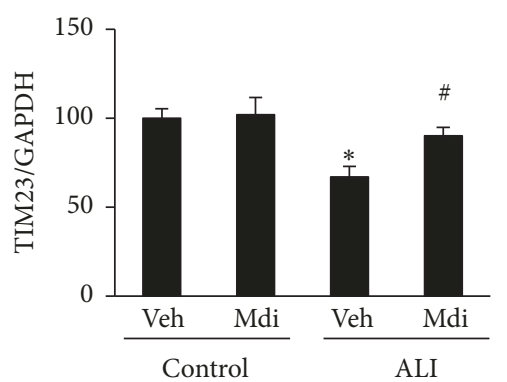

(c)

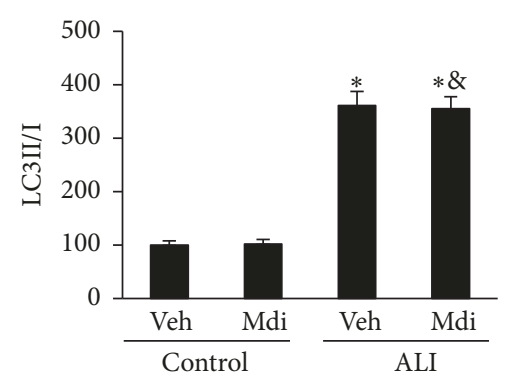

(f)

FIgURE 2: Mdivi-1 treatment inhibits the mitophagy activation. Mdivi-1 treatment prevented the LPS-induced loss of mitochondrial mass without influencing mitochondrial biogenesis and overall autophagy. (a) representative images of western blot results are shown; (b to g) semiquantitative analysis of protein levels. The values are expressed as the mean $\pm \mathrm{SD}(\mathrm{n}=6$ in each group). $* \mathrm{P}<0.05$ versus control group; $\# \mathrm{P}<0.05$ versus LPS + vehicle group; \&P >0.05 versus LPS + vehicle group. TIM23, translocase of inner mitochondrial membrane 23 homolog; PGC-1 $\alpha$ : peroxisome proliferator-activated receptor gamma, coactivator 1 alpha; mt-TFA, mitochondrial transcription factor A.

activity of SOD was highly reduced, whereas the level of MDA and LPO was increased following LPS administration $(\mathrm{P}<$ 0.05 for Control versus LPS + vehicle). However, treatment with Mdivi-1 markedly attenuated the consumption of SOD, as well as MDA and LPO accumulation $(\mathrm{P}<0.05$ for LPS + vehicle versus Mdivi-1; Figure 5), suggesting that Mdivi-1 provides protection against LPS-induced oxidative stress.

3.6. Mdivi-1 Treatment Prevents Inflammation Response in the Lung. Considering the key role of inflammatory mediators in ALI[4], we next measured the level of inflammatory mediators in the lung and BALF. As shown in Figure 6, increased levels of TNF- $\alpha$, IL- $1 \beta$, and IL- 6 in the lungs and BALF of LPS-challenged rats were detected compared to those in the control group $(\mathrm{P}<0.05$ for Control versus LPS). However, Mdivi-1 treatment significantly suppressed the levels of above cytokines in the lung and BALF $(\mathrm{P}<0.05$ for LPS + vehicle versus LPS + Mdivi-1; Figures 6(a)-6(f)). Neutrophilic inflammation is associated with ALI[16]. Therefore, as an indicator of neutrophil infiltration, the lung MPO activity was detected. As shown in Figure 6(g), the lung MPO activity in LPS-challenged rats dramatically increased $(\mathrm{P}<$ 0.05 for Control versus LPS), and it was inhibited by Mdivi-1 treatment $(\mathrm{P}<0.05$ for LPS + vehicle versus LPS + Mdivi1). In addition, the level of F4/80 (indicating macrophages) expression increased following LPS administration $(\mathrm{P}<0.05$ for Control versus LPS + vehicle), and Mdivi-1 treatment inhibited the upregulation of F4/80 expression (Figure 6(i)). 


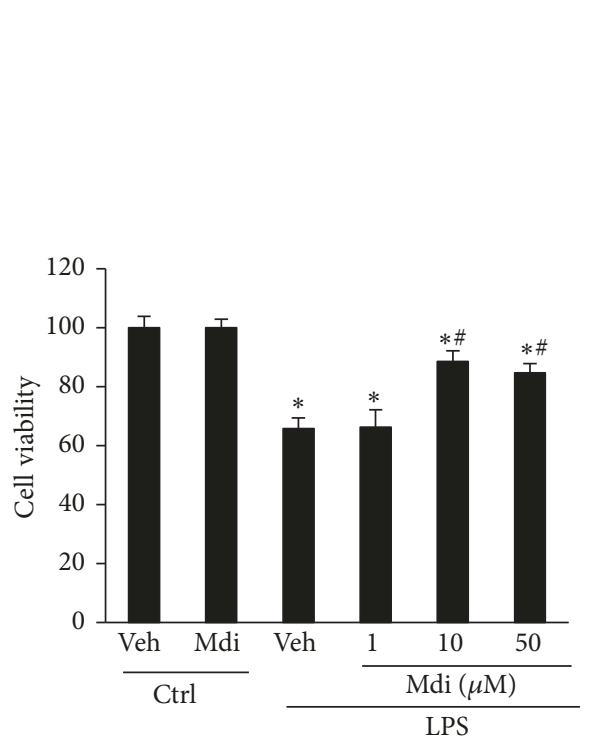

(a)

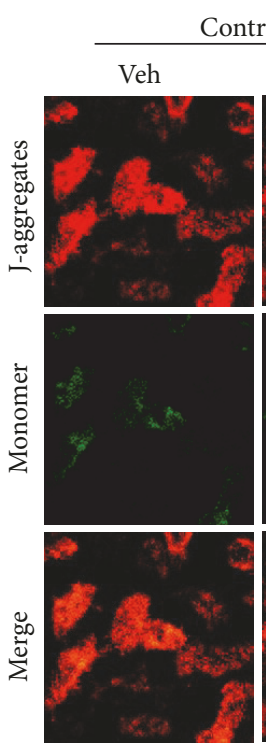

trol
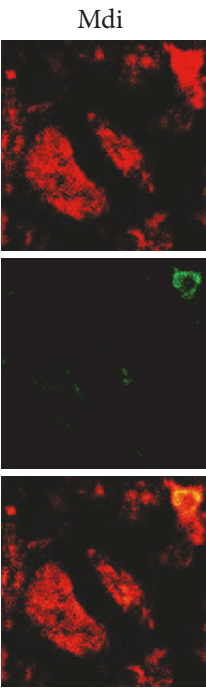

(b)

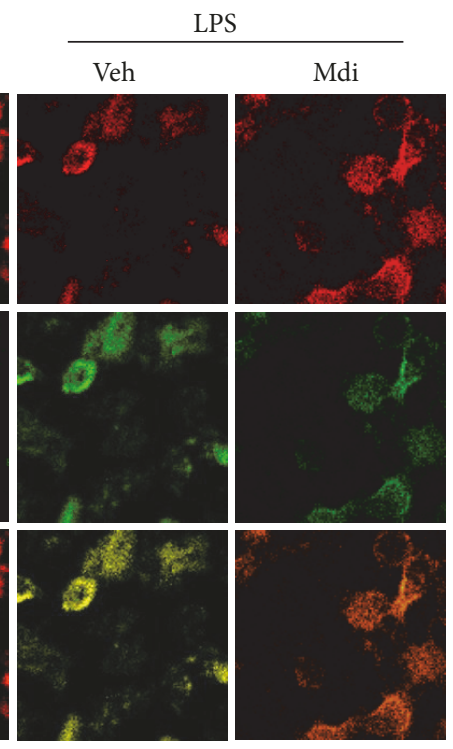

)

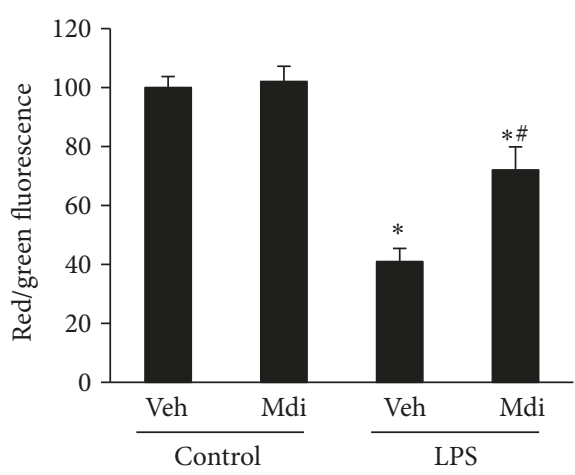

(c)

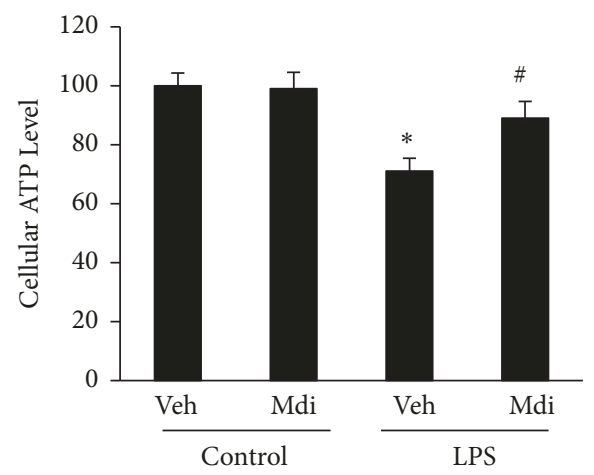

(d)

FIGURE 3: Mdivi-1 treatment inhibits LPS-induced mitochondrial dysfunction in vitro. (a) Quantification of the cells viability: the LPS + vehicle group showed remarkable decreased cell viability. Moderate $(10 \mu \mathrm{M})$ and high $(50 \mu \mathrm{M})$ doses of Mdivi-1 treatment markedly reversed the cell viability induced by LPS exposure. (b) The cells were stained with JC-1 and the mitochondrial membrane potential $(\Delta \Psi \mathrm{m})$ was observed using laser confocal-scanning microscopy. Scale bar: $20 \mu \mathrm{M}$. (c) Quantification of the intracellular red and green fluorescence of JC-1. (d) Quantification of the cellular ATP levels. Data are presented as the mean \pm SD ( $\mathrm{n}=6$ in each group). $* \mathrm{P}<0.05$ versus the control group; $\# \mathrm{P}$ $<0.05$ versus the LPS + vehicle group.

These results suggest that Mdivi-1 treatment downregulates LPS-induced inflammation and resists inflammatory cells (neutrophils and macrophages) recruitment in lung.

\section{Discussion}

In the present study, we show that Mdivi-1 attenuated mitophagy and ALI induced by LPS using a rat model. Moreover, we demonstrated this protective role of Mdivi-1 to be mediated by ameliorating mitochondrial dysfunction, oxidative stress, apoptosis, and inflammation. These findings suggest that mitophagy may be involved in the pathogenesis of ALI, and inhibition of mitophagy may be a potential target for ALI therapy.

As previously described, a rat model of ALI was generated by delivering an intratracheal injection of LPS $(10 \mathrm{mg} / \mathrm{kg})[17-$ 19]. As expected, remarkable lung injury and dysfunction was observed following LPS administration, as evidenced by the level of histopathologic deterioration, elevated W/D weight ratio, and decreased oxygenation index, which is similar to that reported in previous studies $[2,20]$. Then, we investigated the protective effects of different doses of Mdivi1 (low: $1 \mathrm{mg} / \mathrm{kg}$; moderate: $3 \mathrm{mg} / \mathrm{kg}$; high: $5 \mathrm{mg} / \mathrm{kg}$ ) against LPS-induced ALI. Treatment with the moderate and high dose of Mdivi-1 was associated with beneficial effects in LPSinduced ALI, as reflected by an improvement in pathological changes, water content in the lungs, and oxygenation index.

A growing number of reports suggest that mitophagy is relevant to several human diseases. The study by Mizumura et al. found that excessive mitophagy promotes mitochondrial damage in chronic obstructive pulmonary disease [9]. In addition, $\mathrm{Wu}$ et al. showed excessive autophagy and mitophagy activation in blood-brain barrier disruption, cell death, and oxidant stress induced by traumatic 


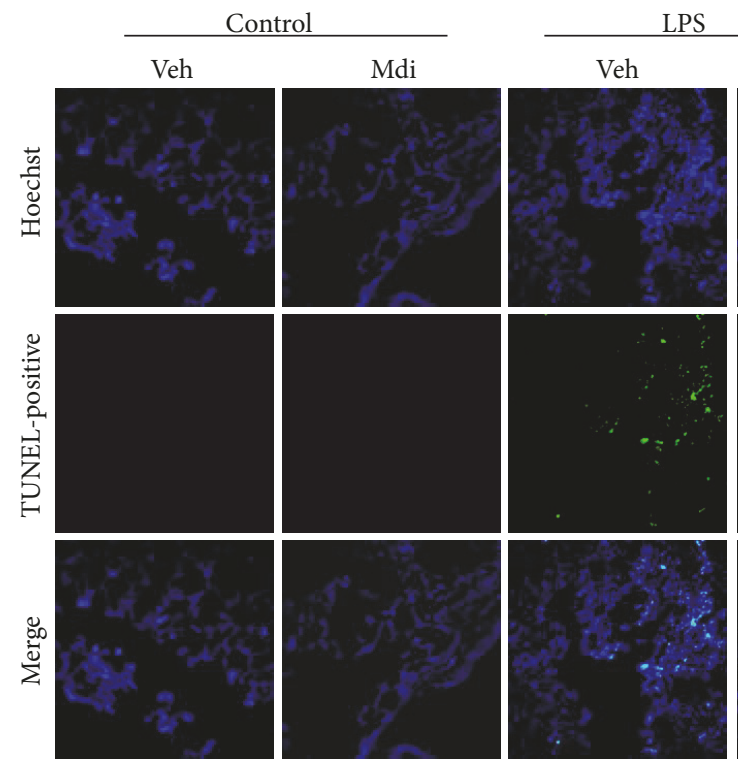

(a)

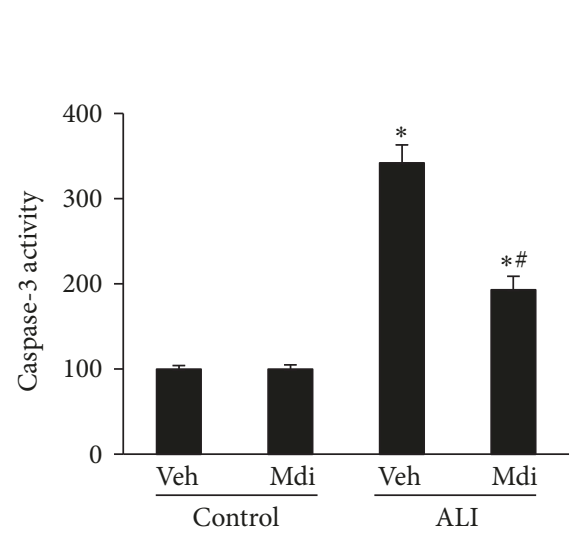

(c)

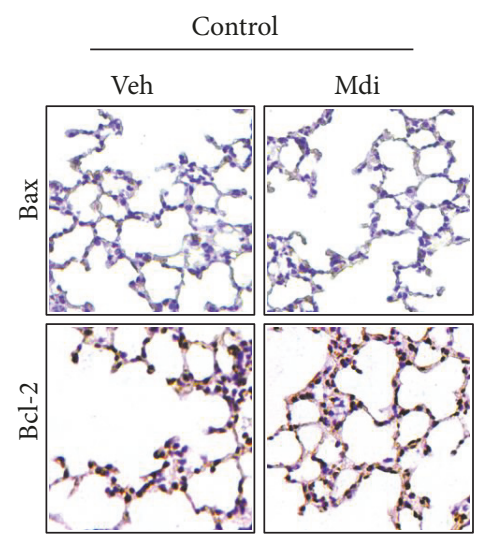

(d)

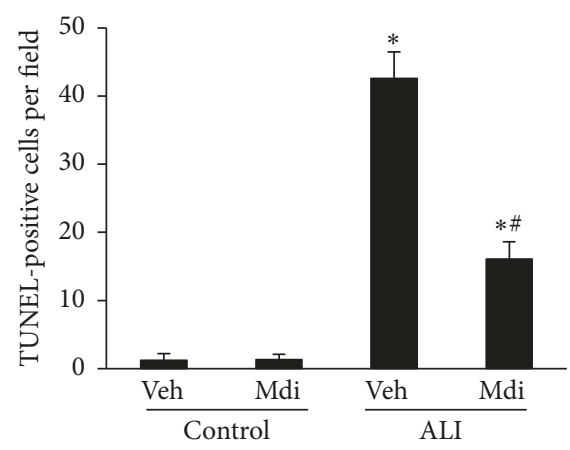

(b)

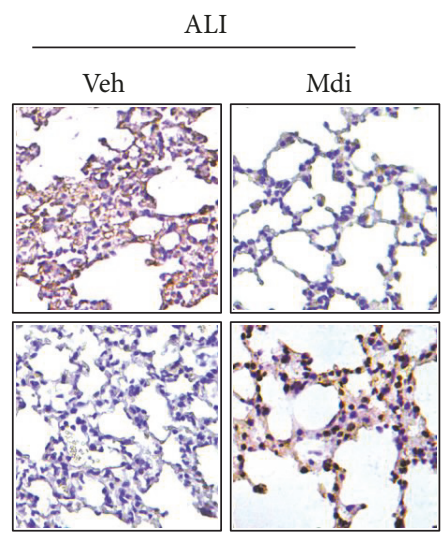

Figure 4: Mdivi-1 prevents lung cell apoptosis in vivo. (a) The level of pulmonary cell apoptosis increased following LPS administration, as determined by TUNEL staining (200× magnification). (b) TUNEL-positive cells were averaged over 10 microscopic fields per animal. LPSchallenged animals exhibited a significant increase in TUNEL-positive cells, which was reduced by treatment with Mdivi-1 treatment. (c) Caspase-3 activity in the lung tissues was elevated in the LPS group but suppressed in the group treated with Mdivi-1. (d) The level of Bax and $\mathrm{Bcl}-2$ protein expression was determined by immunohistochemistry. The increased Bax expression and decreased Bcl-2 expression in the LPS group was reversed following Mdivi-1. Data are presented as the mean $\pm \mathrm{SD}(\mathrm{n}=6$ in each group). $* \mathrm{P}<0.05$ versus control group; \#P $<$ 0.05 versus LPS + vehicle group.

brain injury [21]. While growing evidence indicates that mitophagy may be enhanced in sepsis [9, 22, 23], the role of mitophagy in ALI remains unknown. Mitophagy leads to the phagocytosis of mitochondria, thus decreasing the intracellular mitochondrial mass when it is enhanced [24]. In the present study, we investigated changes in the mitochondrial mass to detect mitophagy. We found that TOM20 and TIM23 levels were decreased in rats with ALI, indicating that mitophagy was upregulated. Previous studies use Mdivi-1, a pharmacological Drpl inhibitor, to investigate the effects of inhibiting mitophagy [25]. Consistent with such studies, we found that Mdivi-1 treatment inhibited the downregulation of TOM 20 and TIM23 expression. In addition, the decrease in mitochondrial mass may result from the reduction of mitochondrial production or an increase in autophagy. We found that the increased LC3 II/LC3 I ratio and decreased p62 expression induced by LPS were not reversed following Mdivi-1 treatment. Moreover, expression of the mitochondrial biogenesis markers, PGC- $1 \alpha$ and mtTFA, was not significantly downregulated following LPS or Mdivi-1 treatment. These findings indicted that Mdivi-1 had no significant effect on mitochondrial production or general autophagy, suggesting that Mdivi-1 affects mitochondrial mass by modulating mitophagy.

Mitochondrial dysfunction is correlated with sepsis, and normal depolarization of the mitochondrial membrane is 


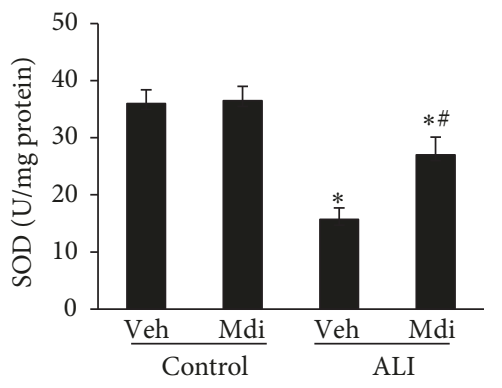

(a)

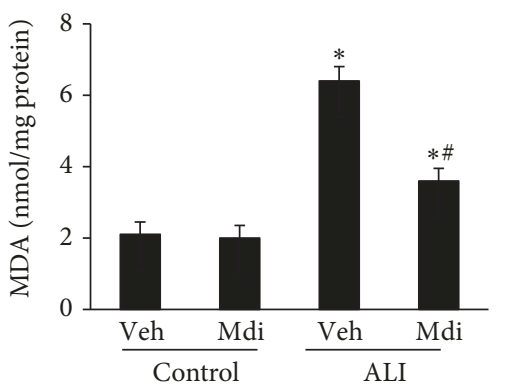

(b)

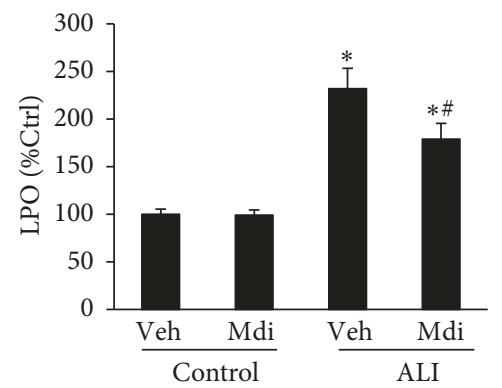

(c)

FIgURE 5: Mdivi-1 ameliorates oxidative stress in pulmonary tissues. (a) LPS administration induced significant SOD consumption, which was attenuated by Mdivi-1 treatment. (b) LPS administration induced significant MDA accumulation, which could be attenuated by Mdivi-1 treatment. (c) LPO levels in the lung increased in the LPS group but decreased in the group that received Mdivi-1. Data are presented as the mean $\pm \mathrm{SD}$ ( $\mathrm{n}=6$ in each group). $* \mathrm{P}<0.05$ versus control group; $\# \mathrm{P}<0.05$ versus LPS + vehicle. SOD, superoxide dismutase; MDA, malondialdehyde; LPO, Lipid peroxides.

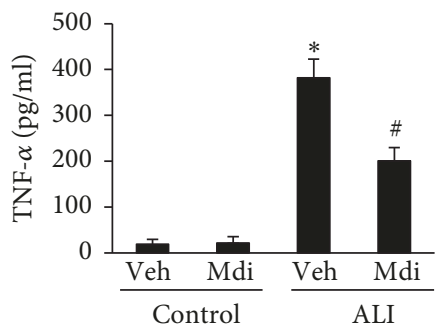

(a)

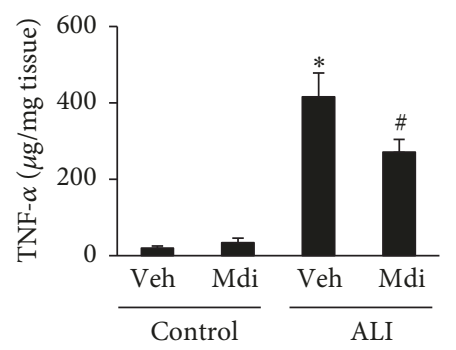

(d)

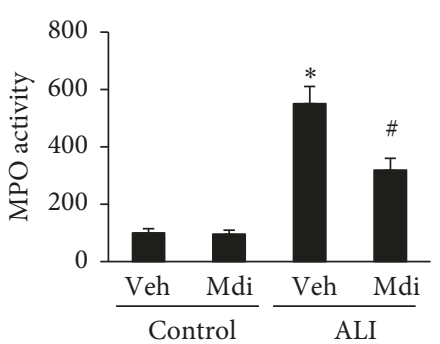

(g)

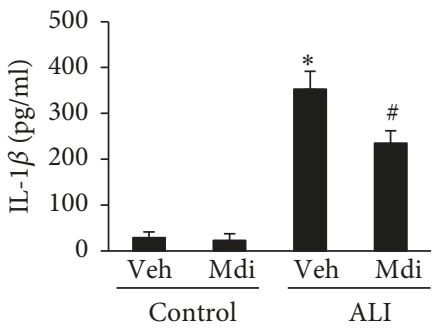

(b)

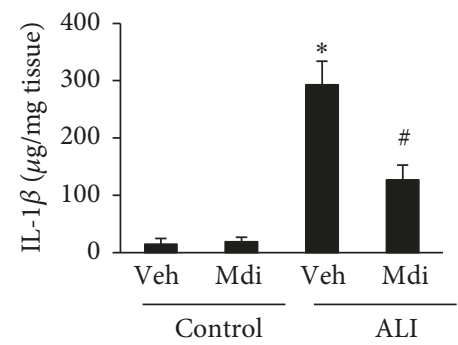

(e)

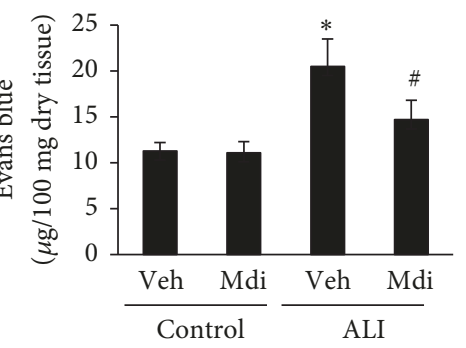

(h)

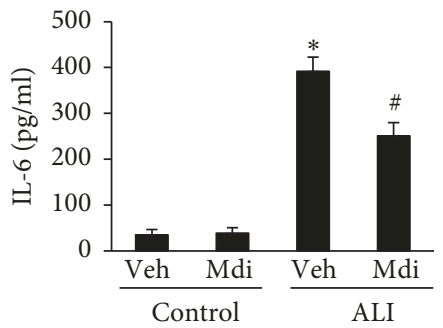

(c)

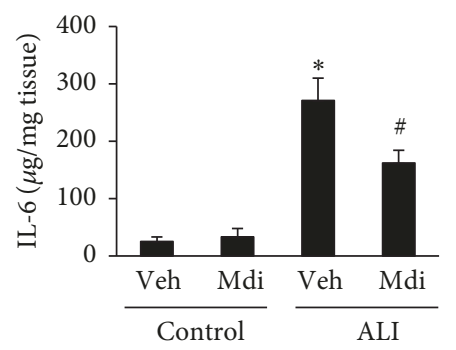

(f)

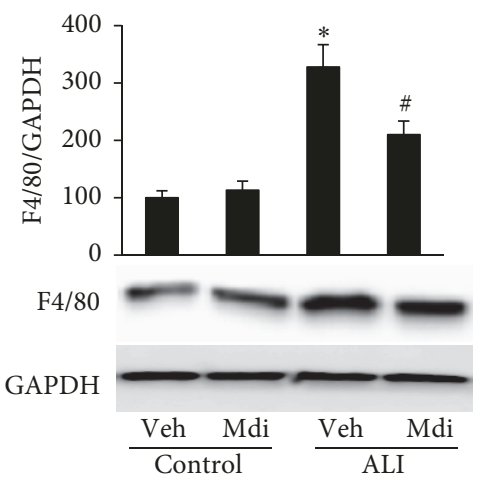

(i)

Figure 6: Mdivi-1 downregulated the levels of proinflammatory cytokines as well as the lung inflammatory cells recruitment. The levels of inflammatory mediators were elevated in the LPS + vehicle group, but decreased with Mdivi-1 treatment group. (a to c) TNF- $\alpha$, IL-1 $\beta$ and IL-6 level in BALF. ( $\mathrm{d}$ to f) TNF- $\alpha$, IL-1 $\beta$ and IL-6 level in lung. (g) Lung MPO activity. (h) Evans Blue (EB) content in lungs. (i) Images of western blot result and semiquantitative analysis of F4/80 level. Data are presented as the mean $\pm \mathrm{SD}(\mathrm{n}=6$ in each group). $* \mathrm{P}<0.05$ versus control group; \#P $<0.05$ versus LPS + vehicle. 
vital to cell survival [26]. Opening of the mitochondrial permeability transition pore (mPTP) causes dissipation of mitochondrial membrane potential $(\Delta \Psi \mathrm{m})$ and inhibition of ATP production and results in mitochondria dysfunction and even cell death. In the current study, decreased cell viability, collapsed $\Delta \Psi \mathrm{m}$, and low intracellular ATP developed in A549 cells with LPS insult, however, Mdivi-1 prevented this mitochondrial injury and cell death in vitro. Several studies have shown that mitochondrial dynamics are linked to mitochondrial quality control by mitophagy, and Mdivi-1 alleviates the mitochondrial damage in traumatic brain injury[21], doxorubicin-induced cardiotoxicity [27], and triptolide-induced hepatotoxicity.[28] Similarly, our results demonstrate that Mdivi-1 treatment attenuated lung injury, indicating that the inhibition of mitophagy may play a protective role in ALI.

Apoptosis is a widely accepted critical pathophysiological process in ALI $[29,30]$. Thus, the results of the present study further demonstrate that Mdivi-1 treatment provided protection in ALI by functioning as an inhibitor of apoptosis. In this study, we observed a significant reduction in the density of apoptotic cells after delivering Mdivi-1 treatment. In addition, the change in the expression of several apoptosis-related proteins (i.e., Bcl-2, Bax, and caspase 3) was assessed. It has been well-established that $\mathrm{Bcl}-2$ is an antiapoptotic protein, whereas Bax is considered to be a proapoptotic member of the Bcl-2 family proteins [31-33]. In our study, Mdivi-1 inhibited the LPS-induced upregulation of Bax downregulation of $\mathrm{Bcl}$ 2 and activation of caspase 3. Collectively, these findings suggest that Mdivi-1 treatment plays an antiapoptosis role in LPS-induced ALI.

Oxidative stress also has a critical role in the pathophysiology associated with sepsis. [8,29] In the present study, superoxide dismutase (SOD) consumption, lipid peroxides (LPO) levels, and malondialdehyde (MDA) accumulation were used as a measure of oxidative stress. Increased LPO levels, MDA accumulation, and SOD consumption in the lung tissues of LPS-challenged rats were all reversed by Mdivi-1 treatment. These results suggest that Mdivi-1 treatment can significantly reduce LPS-induced oxidative stress in ALI.

Proinflammatory cytokines play an essential role in the initiation and amplification of inflammatory responses [34, 35], and recruitment of neutrophils is a key event in development of ALI. The effect of neutrophils is supported by studies where lung injury is reversed by neutrophils consumption $[36,37]$. In the present study, rats exposed to LPS exhibited a significant upregulation of inflammatory mediators and recruitment of inflammatory cells including neutrophils and macrophages; we found that Mdivi-1 treatment dramatically prevented the increase of proinflammatory cytokines (TNF$\alpha$, IL-1 $\beta$, and IL-6) in lung and BALF, MPO activity, and level of F4/80. These results demonstrate that Mdivi-1 treatment ameliorates LPS-induced lung inflammation response.

\section{Conclusion}

The present study demonstrates that the mitophagy inhibitor, Mdivi-1, inhibits mitophagy and protects ALI-challenged rats from LPS-induced apoptosis, oxidative stress, and inflammation. Furthermore, in this study, the wider protection and inhibition of mitophagy provided by Mdivi-1 ( 3 or $5 \mathrm{mg} / \mathrm{kg}$ ) may represent a promising target for the treatment of ALI in the future.

\section{Data Availability}

All data supporting for this study is found in the manuscript or is available upon request from the correspinding author, Youtan Liu.

\section{Disclosure}

$\mathrm{Xu}$ Luo and Ruimeng Liu are equal contributors.

\section{Conflicts of Interest}

The authors declare no finical or commercial conflicts of interest.

\section{Authors' Contributions}

Ruimeng Liu and Xu Luo conducted the study, analyzed the data, and prepared the manuscript. Zhugui Chen and Zhihao Zhang performed the animal experiments and prepared the manuscript. Youtan Liu and Jian He designed the study and reviewed and edited the manuscript. All authors have approved and provided critical input to the final manuscript.

\section{Acknowledgments}

This study was supported by the National Natural Science Foundation of China (81670075) and the Research Foundation of Shenzhen Hospital of Southern Medical University (8167010880).

\section{References}

[1] M. S. Herridge, A. M. Cheung, C. M. Tansey et al., "Oneyear outcomes in survivors of the acute respiratory distress syndrome," The New England Journal of Medicine, vol. 348, no. 8, pp. 683-693, 2003.

[2] K. Xie, Y. Yu, Y. Huang et al., "Molecular hydrogen ameliorates lipopolysaccharide-induced acute lung injury in mice through reducing inflammation and apoptosis," Shock, vol. 37, no. 5, pp. 548-555, 2012.

[3] J. Cohen, "The immunopathogenesis of sepsis," Nature, vol. 420, no. 6917, pp. 885-891, 2002.

[4] T. Zhu, D. X. Wang, W. Zhang et al., "Andrographolide protects against LPS-Induced Acute Lung Injury by Inactivation of NF$\kappa \mathrm{B}, "$ PLoS ONE, vol. 8, no. 2, Article ID e56407, 2013.

[5] P. R. Crisostomo, M. Wang, C. M. Herring et al., "Gender differences in injury induced mesenchymal stem cell apoptosis and VEGF, TNF, IL-6 expression: role of the $55 \mathrm{kDa}$ TNF receptor (TNFR1)," Journal of Molecular and Cellular Cardiology, vol. 42, no. 1, pp. 142-149, 2007. 
[6] A. G. Mustafa, I. N. Singh, J. Wang, K. M. Carrico, and E. D. Hall, "Mitochondrial protection after traumatic brain injury by scavenging lipid peroxyl radicals," Journal of Neurochemistry, vol. 114, no. 1, pp. 271-280, 2010.

[7] Y. Fang, P. Xu, C. Gu et al., "Ulinastatin improves pulmonary function in severe burn-induced acute lung injury by attenuating inflammatory response," Journal of Trauma - Injury Infection and Critical Care, vol. 71, no. 5, pp. 1297-1304, 2011.

[8] M. C. Cimolai, S. Alvarez, C. Bode, and H. Bugger, "Mitochondrial mechanisms in septic cardiomyopathy," International Journal of Molecular Sciences, vol. 16, no. 8, pp. 17763-17778, 2015.

[9] K. Mizumura, S. M. Cloonan, K. Nakahira et al., "Mitophagydependent necroptosis contributes to the pathogenesis of COPD," The Journal of Clinical Investigation, vol. 124, no. 9, pp. 3987-4003, 2014.

[10] A. S. Gonzalez, M. E. Elguero, P. Finocchietto et al., "Abnormal mitochondrial fusion-fission balance contributes to the progression of experimental sepsis," Free Radical Research, vol. 48, no. 7, pp. 769-783, 2014.

[11] S. L. Schmid and V. A. Frolov, "Dynamin: Functional design of a membrane fission catalyst," Annual Review of Cell and Developmental Biology, vol. 27, pp. 79-105, 2011.

[12] G. Twig, A. Elorza, A. J. A. Molina et al., "Fission and selective fusion govern mitochondrial segregation and elimination by autophagy," EMBO Journal, vol. 27, no. 2, pp. 433-446, 2008.

[13] C. Brooks, Q. Wei, S. Cho, and Z. Dong, "Regulation of mitochondrial dynamics in acute kidney injury in cell culture and rodent models," The Journal of Clinical Investigation, vol. 119, no. 5, pp. 1275-1285, 2009.

[14] D. Li, L. Song, J. Wang, C. Meng, and X. Cui, "Adiponectin protects against lung ischemia-reperfusion injury in rats with type 2 diabetes mellitus," Molecular Medicine Reports, vol. 17, pp. 7191-7201, 2018.

[15] G. Cavriani, R. M. Oliveira-Filho, A. G. Trezena et al., "Lung microvascular permeability and neutrophil recruitment are differently regulated by nitric oxide in a rat model of intestinal ischemia-reperfusion," European Journal of Pharmacology, vol. 494, no. 2-3, pp. 241-249, 2004.

[16] Y. Zhang, H. Liu, J. Yao et al., "Manipulating the air-filled zebrafish swim bladder as a neutrophilic inflammation model for acute lung injury," Cell Death \& Disease, vol. 7, no. 11, Article ID e2470, 2016.

[17] J. M. Cavaillon, "Proinflammatory and anti-inflammatory cytokines as mediators of gram-negative sepsis," in Cytokines and Chemokines in Infectious Diseases Handbook. Infectious Disease, M. Kotb and T. Calandra, Eds., Humana Press, Totowa, NJ, USA, 2003.

[18] D. G. Remick and P. A. Ward, "Evaluation of endotoxin models for the study of sepsis," Shock, vol. 24, no. 1, pp. 7-11, 2005.

[19] S. M. Opal, "The host response to endotoxin, antilipopolysaccharide strategies, and the management of severe sepsis," International Journal of Medical Microbiology, vol. 297, no. 5, pp. 365-377, 2007.

[20] H. Lv, Q. Liu, Z. Wen, H. Feng, X. Deng, and X. Ci, "Xanthohumol ameliorates lipopolysaccharide (LPS)-induced acute lung injury via induction of AMPK/GSK3 $\beta$-Nrf2 signal axis," Redox Biology, vol. 12, pp. 311-324, 2017.
[21] Q. Wu, C. Gao, H. Wang et al., "Mdivi-1 alleviates bloodbrain barrier disruption and cell death in experimental traumatic brain injury by mitigating autophagy dysfunction and mitophagy activation," The International Journal of Biochemistry \& Cell Biology, vol. 94, pp. 44-55, 2018.

[22] Q.-P. Liu, D.-X. Zhou, P. Lin, X.-L. Gao, L. Pan, and F.-G. Jin, "Participation of autophagy in acute lung injury induced by seawater," Experimental Lung Research, vol. 39, no. 10, pp. 441452, 2013.

[23] Y. Zhang, G. Liu, R. O. Dull, D. E. Schwartz, and G. Hu, "Autophagy in pulmonary macrophages mediates lung inflammatory injury via NLRP3 inflammasome activation during mechanical ventilation," American Journal of Physiology-Lung Cellular and Molecular Physiology, vol. 307, no. 2, pp. L173-L185, 2014.

[24] Z. Shen, Y. Zheng, J. Wu et al., "PARK2-dependent mitophagy induced by acidic postconditioning protects against focal cerebral ischemia and extends the reperfusion window," Autophagy, vol. 13, no. 3, pp. 473-485, 2017.

[25] M. Zhou, Z. Y. Xia, S. Q. Lei, Y. Leng, and R. Xue, "Role of mitophagy regulated by Parkin/DJ-1 in remote ischemic postconditioning-induced mitigation of focal cerebral ischemia-reperfusion," European Review for Medical and Pharmacological Sciences, vol. 19, pp. 4866-4871, 2015.

[26] G. Garrabou, C. Morén, S. López et al., “The effects of sepsis on mitochondria," The Journal of Infectious Diseases, vol. 205, no. 3, pp. 392-400, 2012.

[27] J. Yin, J. Guo, Q. Zhang et al., "Doxorubicin-induced mitophagy and mitochondrial damage is associated with dysregulation of the PINK1/parkin pathway," Toxicology in Vitro, vol. 51, pp. 1-10, 2018.

[28] M. Hasnat, Z. Yuan, M. Naveed et al., "Drpl-associated mitochondrial dysfunction and mitochondrial autophagy: a novel mechanism in triptolide-induced hepatotoxicity," Cell Biology and Toxicology, 2018.

[29] K. Mantzarlis, V. Tsolaki, and E. Zakynthinos, "Role of oxidative stress and mitochondrial dysfunction in sepsis and potential therapies," Oxidative Medicine and Cellular Longevity, vol. 2017, Article ID 5985209, 10 pages, 2017.

[30] P. Mannam, A. S. Shinn, A. Srivastava et al., "MKK3 regulates mitochondrial biogenesis and mitophagy in sepsis-induced lung injury," American Journal of Physiology-Lung Cellular and Molecular Physiology, vol. 306, no. 7, pp. L604-L619, 2014.

[31] S. Matsuo, W. Yang, M. Aziz, A. Jacob, and P. Wang, "Cyclic arginine-glycine-aspartate attenuates acute lung injury in mice after intestinal ischemia/reperfusion," Critical Care, vol. 17, article R19, 2013.

[32] G. C. Shore and M. Nguyen, "Bcl-2 proteins and apoptosis: choose your partner," Cell, vol. 135, no. 6, pp. 1004-1006, 2008.

[33] P. E. Czabotar, D. Westphal, G. Dewson et al., "Bax crystal structures reveal how $\mathrm{BH} 3$ domains activate $\mathrm{Bax}$ and nucleate its oligomerization to induce apoptosis," Cell, vol. 152, no. 3, pp. 519-531, 2013.

[34] C. Li, J. Liu, L. Zhao, D. Di, M. Meng, and S. Jiang, "Capillary zone electrophoresis for separation and analysis of four diarylheptanoids and an $\alpha$-tetralone derivative in the green walnut husks (Juglans regia L.)," Journal of Pharmaceutical and Biomedical Analysis, vol. 48, no. 3, pp. 749-753, 2008.

[35] I. Lee, C. Dodia, S. Chatterjee, S. I. Feinstein, and A. B. Fisher, "Protection against LPS-induced acute lung injury by 
a mechanism-based inhibitor of NADPH oxidase (type 2)," American Journal of Physiology-Lung Cellular and Molecular Physiology, vol. 306, no. 7, pp. L635-L644, 2014.

[36] S. Hoegl, H. Ehrentraut, K. S. Brodsky et al., "NK cells regulate CXCR2+ neutrophil recruitment during acute lung injury," Journal of Leukocyte Biology, vol. 101, no. 2, pp. 471-480, 2017.

[37] J. Rossaint, J. L. Nadler, K. Ley, and A. Zarbock, “Eliminating or blocking 12/15-lipoxygenase reduces neutrophil recruitment in mouse models of acute lung injury," Critical Care, vol. 16, no. 5, article R166, 2012. 


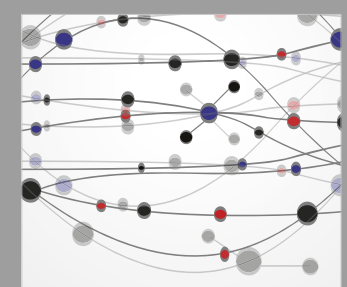

The Scientific World Journal
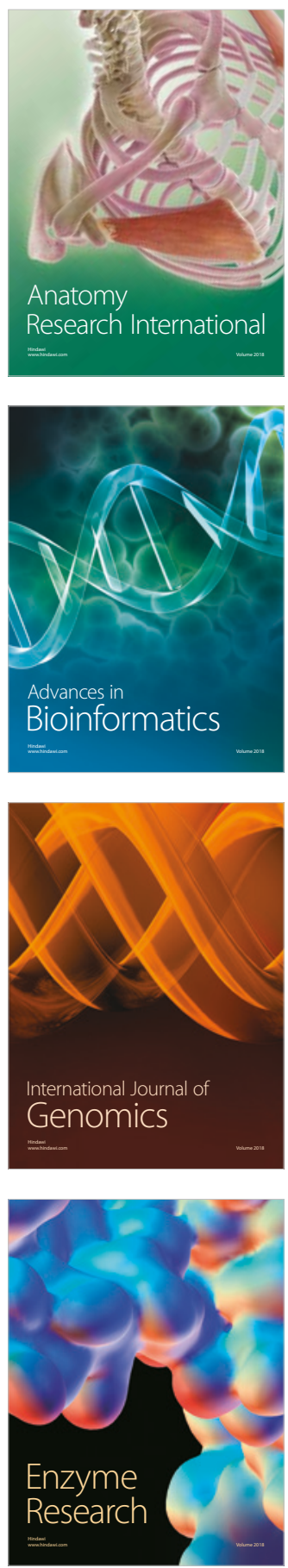
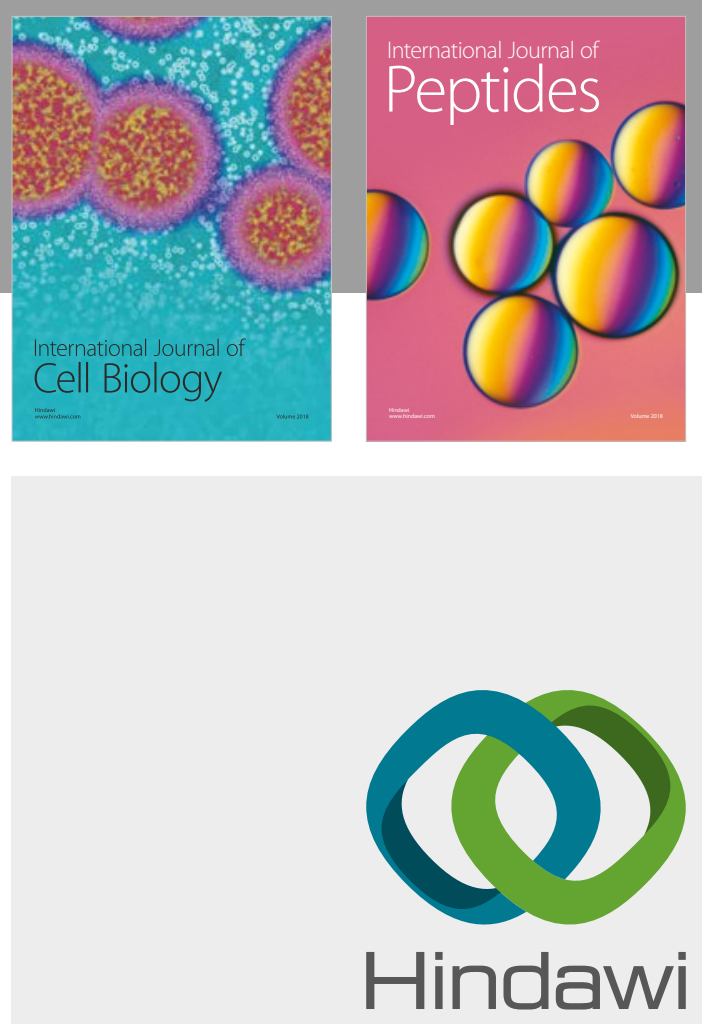

Submit your manuscripts at

www.hindawi.com
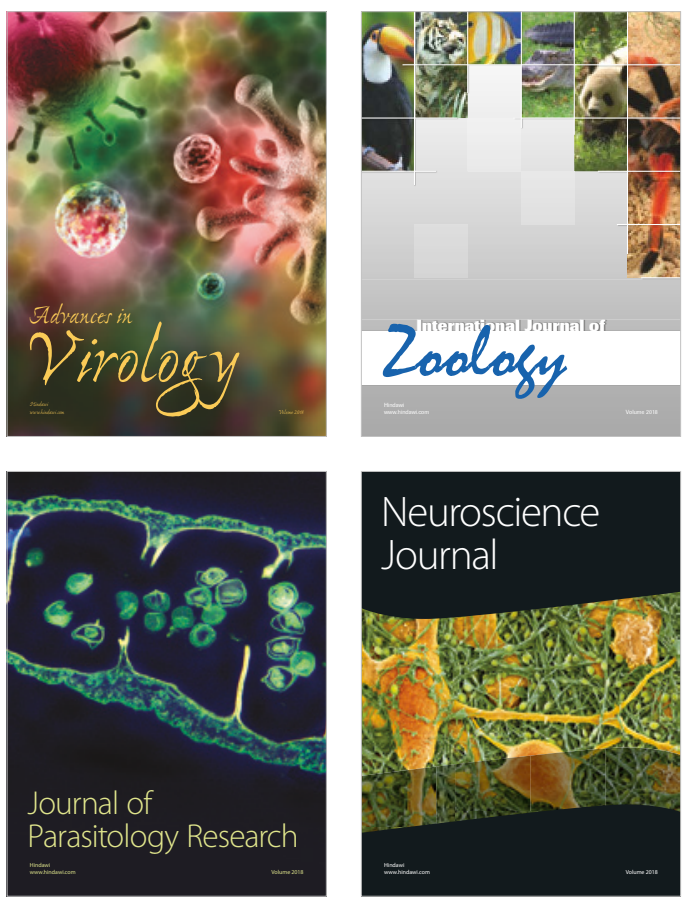
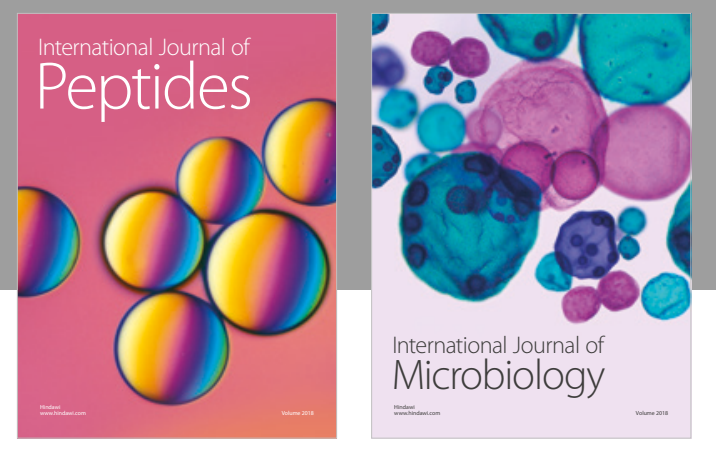

nternational Journal of Microbiology
Journal of
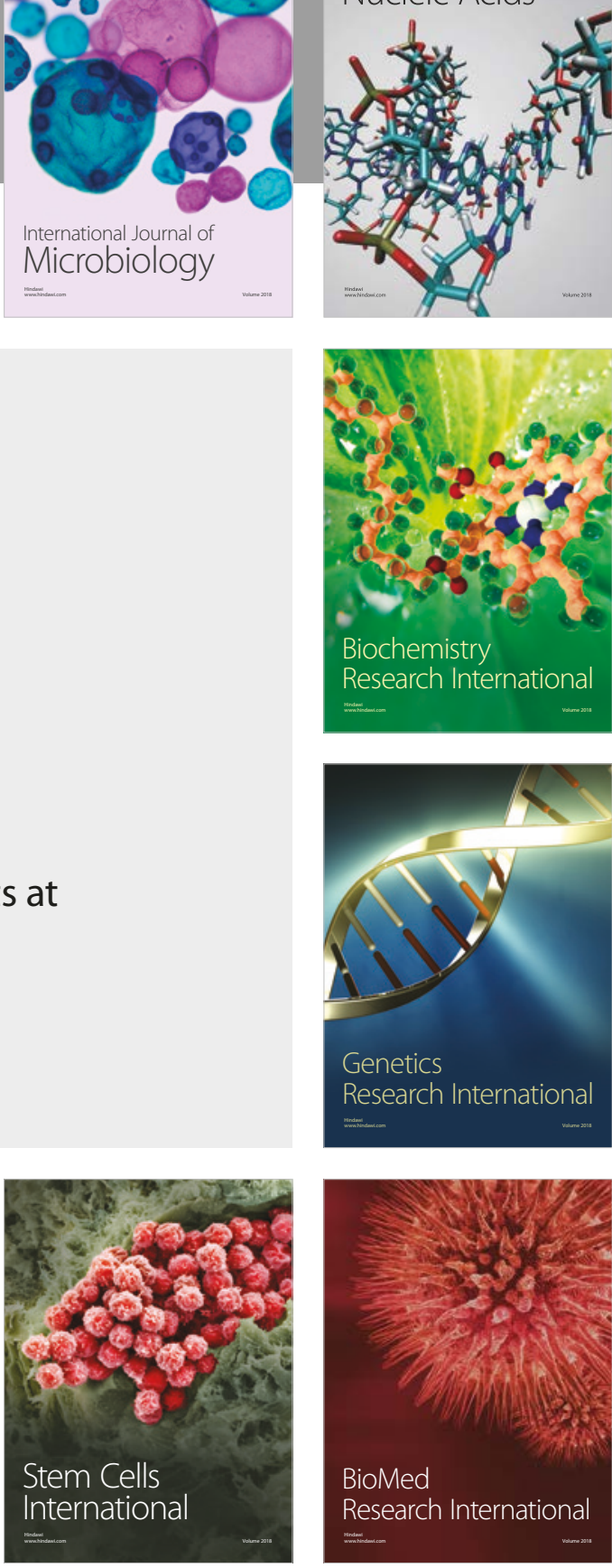
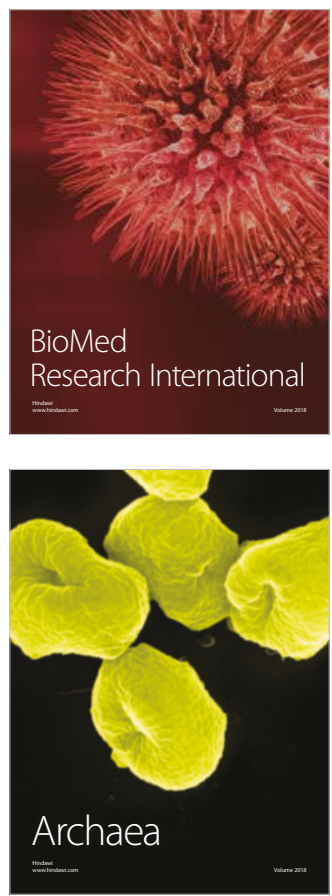